\title{
Single molecule fluorescence spectroscopy: a tool for protein studies approaching cellular environmental conditions $\dagger$
}

\author{
Jörg Fitter, * Alexandros Katranidis, Tobias Rosenkranz, Diaa Atta, Ramona Schlesinger and Georg Büldt*
}

\author{
Received 16th June 2010, Accepted 16th September 2010 \\ DOI: $10.1039 / \mathrm{c} 0 \mathrm{sm} 00538 \mathrm{j}$
}

Due to their high sensitivity and specificity fluorescence based single molecule techniques offer the possibility to study individual molecules (e.g., proteins or protein complexes) in situ in their cellular context. Recent progress in instrumentation and in sample preparation provides an increasingly better accessibility to more complex molecular assemblies. These assemblies mimic the natural cellular environmental conditions and at the same time allow sophisticated studies on proteins of interest. This review gives a brief introduction to single molecule fluorescence techniques and presents some selected applications on protein folding and on complex formation of membrane proteins.

\section{Introduction}

One of the major goals in biology is to understand the function of proteins and of macromolecular complexes in their cellular context. In order to reach this goal, different and typically complementary measuring techniques have to be applied. Fluorescence based methods offer the possibility to measure protein properties and interactions with a high sensitivity and selectivity. The advent of bright and more photostable fluorescent dyes and an enormous methodical and technical improvement of high resolution fluorescence spectroscopy and microscopy enabled studies on proteins even at a single molecule level. ${ }^{1-7}$ If one wants to measure sample parameters in ensemble, the investigated processes have to be synchronized which is often difficult or some times even impossible to achieve. By employing single molecule studies asynchronous processes (e.g., protein folding) can be studied in more detail. The advantage of this technique is given by the fact that it provides information on the distribution of parameters characterizing the protein. From bulk measurements only mean values of these parameters can be extracted..$^{5,8-10}$ The most straightforward approach to study proteins in the cellular context is to measure them directly inside (living) cells. Because most proteins in cells are very mobile and able to diffuse around in restricted areas or compartments within the cell, single particle tracking (SPT) is often employed. This method allows to following individual molecules with high spatial resolution in real time. Numerous interesting cellular aspects have been studied in living cells, which include vesicle trafficking, ${ }^{11}$ nuclear pore transport, ${ }^{12}$ viral infection, ${ }^{13}$ cell membranes and cell signaling, ${ }^{14,15}$ and gene transcription and translation. ${ }^{16}$ In addition various techniques have been developed recently that aim to achieve subdiffraction-resolution in imaging living cells. Although these techniques do not necessarily focus on studying single biomolecules, they make use of the single fluorophore sensitivity to achieve an experimental resolution of $20 \mathrm{~nm}$ or better, which is a tremendous improvement with respect to

Research Centre Jülich, ISB-2: Molecular Biophysics, D-52425 Jülich, Germany.E-mail: g.bueldt@fz-juelich.de; j.fitter@fz-juelich.de

$\dagger$ This paper is part of a Soft Matter themed issue dedicated to the International Soft Matter Conference 2010. conventional imaging techniques. ${ }^{17-21}$ However, although single molecule fluorescence study on proteins in living cells is a powerful approach, it is also often limited by the assortment of fluorescent dyes, by the accessibility of the target molecule, and by the ability to manipulate the sample.

Another promising approach is to study proteins outside the cell in an environment which mimics relevant features of the cell. In the case of single molecule methods these (in vitro) assays typically consist of a low concentration of immobilized proteins or protein-complexes performing their biological activity, for example by interacting with their corresponding binding partners. By choosing a proper labeling configuration, conformational changes of the protein of interest can be monitored in an ongoing biochemical process. An increasing number of interesting and promising results demonstrate that single molecule fluorescence methods in combination with in situ measurements on complex sample arrangements provide a powerful tool to elucidate functional details of proteins and biological nanomachines (see for example ref. 6, 7 and 10 and references therein). In this review we briefly describe the methodology and the technical realization of single molecule fluorescence studies with in vitro assays. We present and discuss results from some recent studies, which made use of the described approach. Since this review is presenting only a few examples focusing on applications with in vitro assays, we refer the readers for an extended overview on methods and applications of fluorescence single molecule studies to other distinguished and more elaborated reviews. ${ }^{5-7,22-25}$

\section{Microscopy and samples}

In order to monitor the properties of individual proteins by fluorescence we need to label the protein of interest with a highly sensitive fluorescent dye (or with several dyes of different colors). In addition we have to keep the protein at a low concentration to make sure that signals from individual probes can be separated. For single molecule fluorescence studies essentially two different types of setups are established. In a typical wide-field fluorescence microscope (Fig. 1A) excitation light from a continuous wave laser is reflected by a (multiband) dichroic mirror into a high numerical aperture objective. Fluorescence emission light from surface tethered fluorescently labeled biomolecules or from 


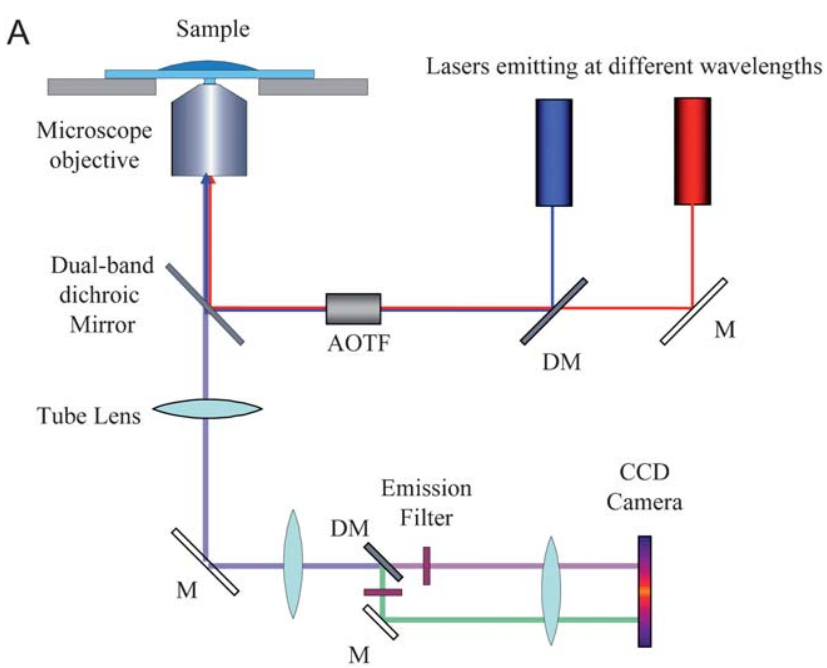

B

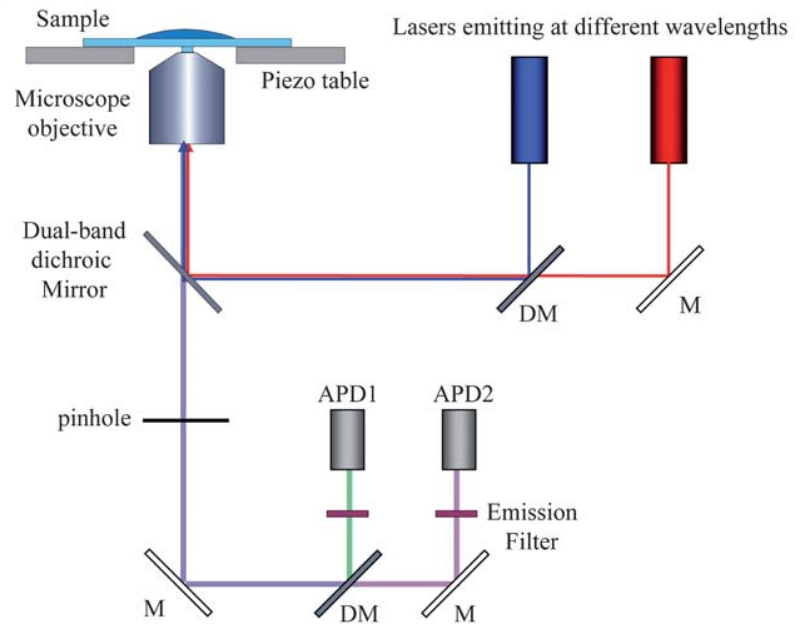

Fig. 1 The simple scheme with the main optical elements (DM: dichroic mirror; M: mirror; AOTF: acousto-optic tunable filter) for the two major high resolution fluorescence microscope setups for dual color detection, (A) a wide-field microscope and (B) a confocal microscope.

fluorophores in immobilized cells is collected by the same objective and passed onto a high-sensitive Peltier-cooled charge coupled device (CCD) camera. For many applications (e.g., colocalization of different molecules, Förster resonance energy transfer, FRET) a setup with at least two colors, which are measured simultaneously, is required. In a dual-color wide-field setup the image of both colors is split by a further dichroic mirror and finally projected into two separated areas of the CCD camera. ${ }^{5,26,27}$ This setup allows the observation of many (up to a few hundred) immobilized individual molecules at the same time. The time resolution for taking an image with this setup is technically limited by the frame rate (up to $100 \mathrm{~Hz}$ ) of the CCD camera. Practically the time resolution is also limited by the molecular brightness of the fluorescent dyes, by the detection efficiency, and by the excitation power. A further useful improvement of this setup is to employ the TIRF (total internal reflection fluorescence) illumination. In contrast to the classical wide-field illumination, TIRF-illumination can yield a much higher signal-to-background ratio (for details see ref. 26 and 28).
Another type of setup is given by a confocal microscope (Fig. 1B). Here the excitation light is tightly focused to a diffraction-limited focal spot that limits the area on the surface (or the volume in solution) of fluorescence detection. The emitted fluorescence light is focused onto a pinhole acting as a small aperture for spatial filtering. After a further dichroic mirror and respective emission filters the light is detected by two avalanche photodiodes (APDs). In contrast to CCD cameras in the widefield setup, APDs offer a much higher time resolution which is sufficient to measure fluorescence life times (ns) if pulsed lasers are employed. In the case of surface tethered molecules this setup can be used as a confocal laser scanning microscope for imaging two colors simultaneously. Compared to the wide-field setup using CCD cameras, the image acquisition by scanning over a surface takes much more time (in the order of tens of seconds). ${ }^{4}$ While typically for the wide-field setup signals can be obtained only from surface immobilized molecules, the confocal microscope can also be used for studies of molecules diffusing freely in solution. This property enables further interesting applications such as fluorescence correlation spectroscopy (FCS) ${ }^{4,29,30}$ or two color single molecule coincidence fluorescence spectroscopy. ${ }^{31}$ For single molecule studies on proteins both setups have advantages and disadvantages. Therefore the employment of both, partly complementary, techniques is very useful in practice.

To study proteins as close as possible in their cellular environment, surface immobilized proteins have the following advantages. (1) Surface immobilization of biomolecules is necessary if the wide-field setup is employed (see above). (2) Individual freely diffusing molecules allow only for limited

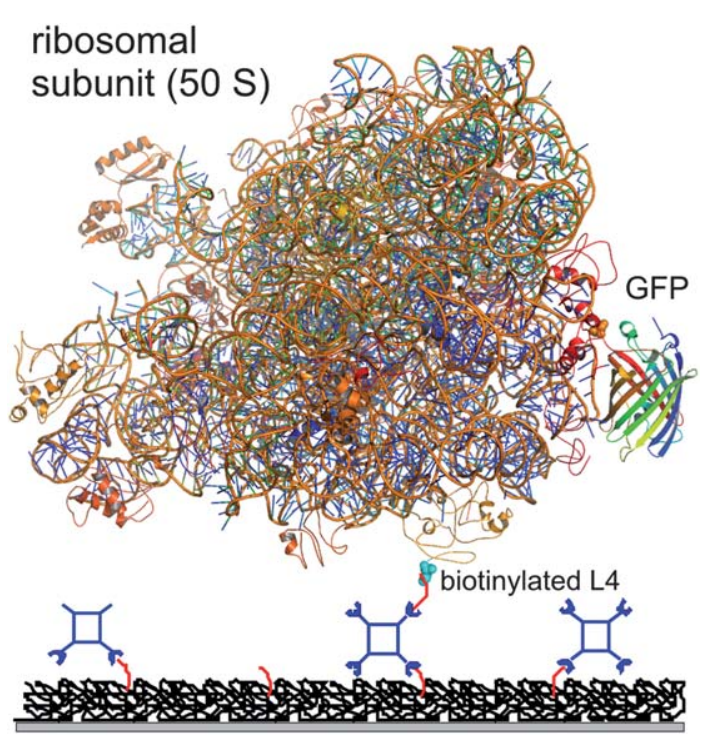

Fig. 2 Schematic view of a $50 \mathrm{~S}$ subunit of ribosome tethered to the surface of a cover slide. The amino-functionalized cover slide is coated with a layer of PEG which is biotinylated at low concentration. By the use of a streptavidin-biotin binding assay fluorescently labeled ribosomes were linked to the surface via biotinylated ribosomal protein L4 (displayed molecules are not on scale). The C-terminal extension of 31 amino-acids of a GFP mutant and the suppression of posttranslational protein release provides the possibility to observe cell-free synthesized GFP which becomes mature while linked to the fluorescence labeled ribosome (reprint with permission from ref. 27). 

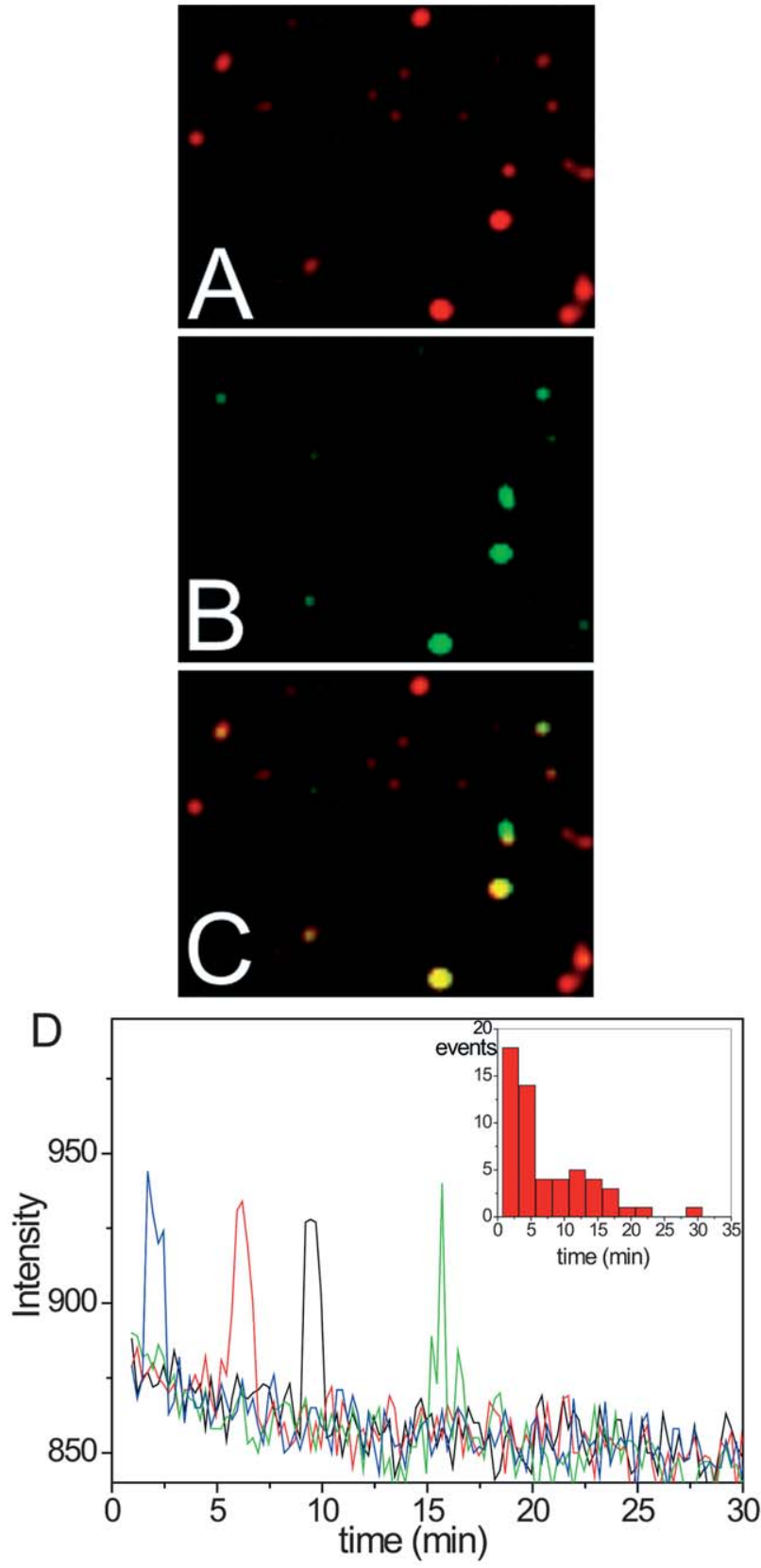

Fig. 3 Selected areas of fluorescence wide-field images as measured for the red emission of labeled ribosomes (A), for the green emission of GFP fluorescence (B), and the overlay of both channels (C) that demonstrates that single surface-tethered ribosomes synthesized GFP molecules which become mature (i.e. fluorescent) while bound to the ribosome. The yellow colored peaks in this figure localize the coexistence of single ribosomes and single GFP molecules bound to their synthesizing ribosomes. (D) Here integrated peak intensities are shown as a function of time for fluorescent GFP molecules appearing at different times after the initiation of biosynthesis. Fluorescence of individual GFP molecules can only be detected for a few consecutive exposures before photobleaching occurs. The inset is showing a histogram with the number of de novo synthesized GFP molecules that appear in consecutive time intervals (reprint with permission from ref. 27).

observation times in the order of milliseconds, which is the diffusion time through the confocal detection volume. Surface immobilized molecules can be monitored for much longer observation times, effectively limited by the photostability of the fluorescent dye. (3) The immobilization of the biomolecules of interest allows an exchange of buffer solutions for initiating or stopping a biochemical process, which in a closed chamber system enables time resolved in situ measurements. ${ }^{27}$ In order to image surface immobilized and functional biomolecules the binding mechanism has to be specific. Unspecific binding must be suppressed as much as possible. Typically the surface of a cover slide is first blocked with polyethylene glycol (PEG) and the specific surface tethering is accomplished by a biotin-streptavidin binding assay (Fig. 2). This approach enables a homogenous surface coverage of functional and labeled biomolecules with a density of a few hundred molecules in an area of about $50 \times$ $50 \mu^{2}$ (for more details see ref. 24 and 26).

\section{Monitoring protein synthesis and protein folding}

In the cell protein synthesis and also folding of proteins take place on ribosomes. In order to elucidate details of these processes several single molecule fluorescence studies with surface tethered ribosomes in action have been performed in the last few years. ${ }^{27,32-34}$ One major focus is to understand how polypeptide chain elongation and folding are coupled. For this purpose de novo synthesis and folding of a green fluorescent protein mutant (GFP Emerald) was observed at single molecule level (Fig. 2 and 3). For imaging fluorescently labeled ribosomes (red detection channel) and emerging GFP molecules (green detection channel) a dual color fluorescence wide-field microscope was used. ${ }^{27}$ The obtained images indicate that approximately $10-15 \%$ of all visible ribosomes produce a bound, mature, and fluorescent GFP (Fig. 3A-C). In a next series of measurements the appearance of individual synthesized GFP molecules was monitored as a function of time (Fig. 3D). The formation of the fluorescent chromophore is a rather slow posttranslational autocatalytic process which often requires at least several minutes. GFP fluorescence shows up with a significant fraction within five minutes after initiating protein synthesis (Fig. 3D). The time course of emerging fluorescent GFP molecules is satisfactorily fitted by a single exponential. The corresponding characteristic time constant for the observed process is 5.3 minutes, which is one of the fastest maturation times for a GFP mutant observed so far. Individual GFP molecules show fluorescence even within one minute (Fig. 3D). Studies aiming to resolve more details on cotranslational folding events require a much better time resolution. For this purpose the maturation of GFP is too slow and a better time resolution for monitoring folding events might be achieved by the cotranslational incorporation of non-natural fluorescent amino acids into the nascent chain. ${ }^{35,36}$

Due to the immobilization of the target molecule rare or slow dynamic events as well as repetitive processes at one and the same molecule can be monitored. An alternative to anchoring proteins directly to a polymer-coated surface with a specific single-point attachment is possible by encapsulating proteins in surfacetethered lipidic, or polymeric vesicles (100-150 $\mathrm{nm}$ in diameter). One requirement for almost all single molecule studies with immobilized proteins is to reduce, as much as possible, interactions of the protein with surfaces. Perturbing interactions of unfolded proteins may significantly alter the structure and the 

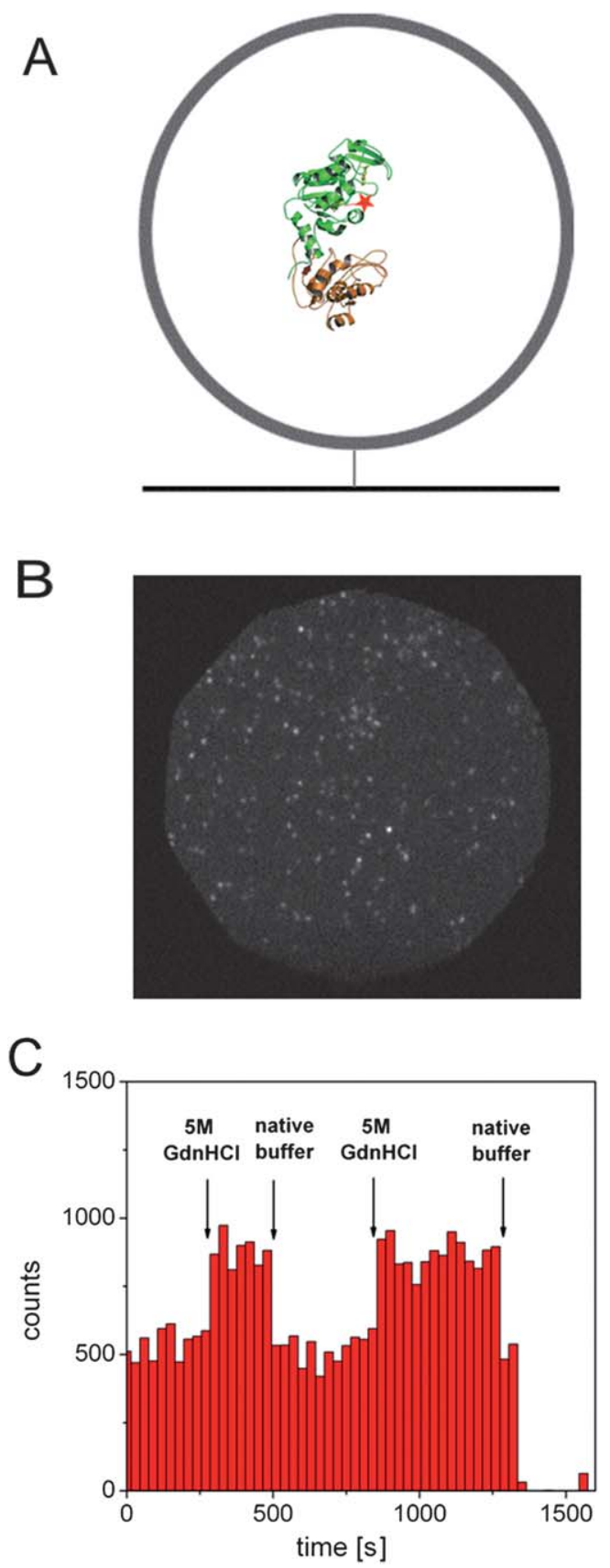

Fig. 4 (A) Scheme of an individual protein labeled with a fluorescent dye encapsulated in a polymerosome. (B) Wide-field fluorescence image of surface-tethered polymerosomes containing dye labeled PGK. (C) A typical time course of the measured emission intensity as obtained from the integration of an individual spot. The corresponding images (see B) were measured every 30 seconds with polymerosomes bound to cover slides which were built-in a closed imaging chamber suitable for in-situ buffer exchange. The arrows indicate buffer exchange from native to unfolding conditions or vice versa (reprint with permission from ref. 41).

dynamics of the protein and can give rise to artifacts in the obtained results. Protein encapsulation is a promising approach to solve this problem by immobilizing water-soluble proteins within a native-like environment. ${ }^{37-41}$ Results of a study with a multi domain protein phosphoglycerate kinase (PGK) encapsulated in surface tethered polymeric vesicles made of amphiphilic triblock copolymers demonstrated the feasibility of this approach ${ }^{41}$ (Fig. 4A). Polymerosomes possess an extreme stability against various chemical denaturants and prove to be permeable to guanidine hydrochloride $(\mathrm{GdnHCl})$ and are thereby ideally suited for unfolding and refolding studies. This was demonstrated with encapsulated PGK, which was fluorescently labeled with Atto655, a dye that exhibits pronounced photoinduced electron transfer (PET) to a nearby tryptophan residue in the native state. ${ }^{42,43}$ Under unfolding conditions with structurally expanded protein structures, PET was reduced. For individual encapsulated proteins alternating unfolding (high fluorescence level) and refolding (low fluorescence level) conditions were monitored (Fig. 4B and C). As an extension of this approach, channel proteins incorporated into the polymeric membrane can facilitate transfer of solutes and substrates across the polymerosome membrane in a controlled manner. ${ }^{44,45}$ Similar investigations have been performed with liposomes. ${ }^{46}$ Channelequipped polymeric nanocontainers extend the range of interesting applications, for example studies of conformational changes localized in immobilized proteins during the interaction with substrate molecules.

\section{Membrane protein interactions studied in lipid bilayers}

The cell membrane is not only a physical barrier between intracellular compartments or against the extracellular environment, but also comprises diverse cellular functions. Thirty percent of all proteins are membrane proteins which act as receptors, pumps, or channels. They facilitate metabolism and communication between cells. To fulfill these functions often larger oligomeric protein complexes have to be formed in the cell membrane or cytosolic proteins have to be anchored to the membrane. Studying protein-complex formation directly in the cell is difficult. Therefore, studies can be performed with giant unilamellar lipid vesicles (GUVs) which mimic a cell membrane to a certain extent. ${ }^{47,48}$ GUVs are characteristically large in size ( $\sim 10$ to $40 \mu \mathrm{m})$ and allow studies at extreme low protein concentrations with special applications of confocal microscopy (Fig. 5A). For the incorporation of functional membrane proteins into GUVs, proteoliposomes with labeled membrane proteins were fused with surface-tethered GUVs. ${ }^{49,50}$ With this procedure a very low protein concentration can be achieved within the GUV, for example a few hundred protein molecules per vesicle, which is rather similar to copy numbers of receptor species in the cell membrane. ${ }^{51}$ In a case study, the lateral diffusion of a photoreceptor with seven transmembrane helices and its cognate transducer (two transmembrane helices) was analyzed in GUVs by employing $\mathrm{FCS}^{52}$ In order to obtain precise and reproducible measurements of diffusion coefficients in GUVs with FCS the central position of the intersecting beam focus is crucial (Fig. 5A). The two-dimensional diffusion coefficients of both separately diffusing proteins (photoreceptor: SRII and the transducer: HtrII) differ significantly (Fig. 5B). In GUVs containing both membrane proteins, a significantly smaller diffusion coefficient was observed for labeled transducer molecules indicating complex formation of SRII with HtrII (HtrII/SRII in Fig. 5B). Based on the phenomenological dependence of diffusion coefficients on the molecule's cylindrical radius, the degree of complex formation can be estimated on a quantitative level. In 


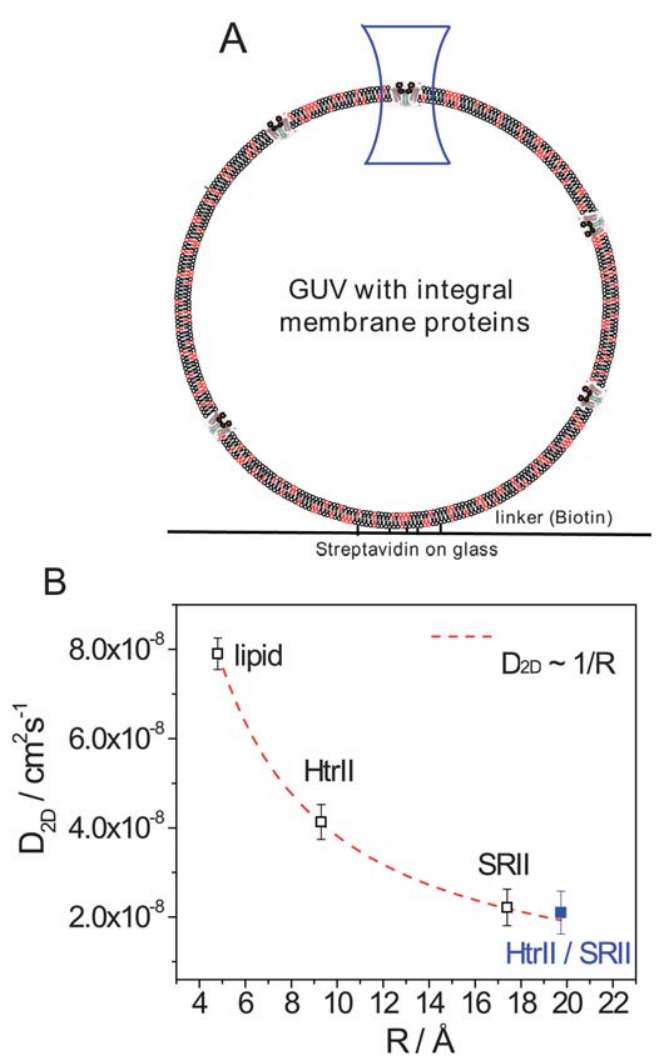

Fig. 5 (A) Schematic side view of a surface tethered GUV with a beam focus (blue colored element) intersecting the GUV membrane at a central vertical focus position. (B) Two-dimensional diffusion coefficients are given as a function of the cylindrical radius of a lipid molecule, the transducer (HtrII), and the receptor (SRII). The dependence of the lateral diffusion on the cylindrical radius $R$ was fitted with a $1 / R$ proportionality (red dotted line) for the two membrane proteins. The blue symbol represents diffusion coefficients of labeled HtrII in the presence of nonlabeled SRII molecules where approximately $80 \%$ of HtrII molecules are bound to an SRII molecule (reprint with permission from ref. 52).

principle this approach is not limited to diffusion related measurements. The degree of protein-complex formation can also be analyzed by two-color cross correlation or by FRET measurements. ${ }^{53}$

\section{Conclusion}

In this review we demonstrated that single molecule fluorescence techniques in combination with easily tunable in vitro assays offer a powerful approach to study protein folding as well as protein complex formation. Since this research field is rapidly developing it is expected that further improvements, in dye labeling, in sample preparations, and in instrumentation are going on. To support this point of view we mention here one further example which emphasizes the interdisciplinary character of this research field. One major limitation in single molecule fluorescence studies is given by the fact that the presence of physiological concentrations of labeled substrates (well above $1 \mu \mathrm{M}$ ) causes too high background fluorescence, even in TIRF illumination. The use of zero-mode waveguides (ZMW) drastically reduces the background signal from freely diffusing fluorescent molecules and allows studies at almost physiological concentration, as nicely demonstrated in some recent applications. ${ }^{54-56}$

\section{Acknowledgements}

We thank our collaborators K. Nierhaus, T. Choli-Papadopoulou, M. Gerrits, I. Gregor, J. Enderlein, W. Meier, and J. Kriegsmann. TR acknowledges financial support by the International Helmholtz Research School on Biophysics and Soft Matter ("BioSoft").

\section{References}

1 T. Ha, T. Enderle, D. F. Ogletree, D. S. Chemla, P. R. Selvin and S. Weiss, Proc. Natl. Acad. Sci. U. S. A., 1996, 93, 6264-6268.

2 H. P. Lu, L. Xun and X. S. Xie, Science, 1998, 282, 1877-1882.

3 M. Dahan, A. A. Deniz, T. J. Ha, D. S. Chemla, P. G. Schultz and S. Weiss, Chem. Phys., 1999, 247, 85-106.

4 M. Bohmer and J. Enderlein, ChemPhysChem, 2003, 4, 793-808.

5 X. Michalet, S. Weiss and M. Jager, Chem. Rev., 2006, 106, 17851813.

6 A. A. Deniz, S. Mukhopadhyay and E. A. Lemke, J. R. Soc., Interface, 2008, 5, 15-45.

7 C. Joo, H. Balci, Y. Ishitsuka, C. Buranachai and T. Ha, Annu. Rev. Biochem, 2008, 77, 51-76.

8 G. Haran, J. Phys.: Condens. Matter, 2003, 15, R1291-R1317.

9 B. Schuler, ChemPhysChem, 2005, 6, 1206-1220.

10 A. N. Kapanidis and T. Strick, Trends Biochem Sci., 2009, 34, 234 243.

11 D. Zenisek, J. A. Steyer and W. Almers, Nature, 2000, 406, 849-854.

12 U. Kubitscheck, D. Grunwald, A. Hoekstra, D. Rohleder, T. Kues, J. P. Siebrasse and R. Peters, J. Cell Biol., 2005, 168, 233-243.

13 G. Seisenberger, M. U. Ried, T. Endress, H. Buning, M. Hallek and C. Brauchle, Science, 2001, 294, 1929-1932.

14 G. J. Schutz, G. Kada, V. P. Pastushenko and H. Schindler, EMBO J., 2000, 19, 892-901.

15 P. H. Lommerse, B. E. Snaar-Jagalska, H. P. Spaink and T. Schmidt, J. Cell Sci., 2005, 118, 1799-1809.

16 J. Yu, J. Xiao, X. Ren, K. Lao and X. S. Xie, Science, 2006, 311, $1600-1603$.

17 S. W. Hell, Nat. Biotechnol., 2003, 21, 1347-1355.

18 E. Betzig, G. H. Patterson, R. Sougrat, O. W. Lindwasser, S. Olenych, J. S. Bonifacino, M. W. Davidson, J. Lippincott-Schwartz and H. F. Hess, Science, 2006, 313, 1642-1645.

19 M. Bates, B. Huang, G. T. Dempsey and X. Zhuang, Science, 2007, 317, 1749-1753.

20 M. Heilemann, L. S. van de, M. Schuttpelz, R. Kasper, B. Seefeldt, A. Mukherjee, P. Tinnefeld and M. Sauer, Angew. Chem., Int. Ed., 2008, 47, 6172-6176.

21 M. Heilemann, P. Dedecker, J. Hofkens and M. Sauer, Laser Photonics Rev., 2009, 3, 180-202.

22 M. Dahan, A. A. Deniz, T. Ha, J. R. Grunwell, D. S. Chemla, P. C. Schultz and S. Weiss, Biophys. J., 1999, 76, A168.

23 S. Weiss, Science, 1999, 283, 1676-1683.

24 T. Ha, P. R. Selvin, Single-molecule techniques, Cold Spring Harbor Laboratory Press, New York, 2008.

25 E. Sisamakis, A. Valeri, S. Kalinin, P. J. Rothwell and C. A. Seidel, Methods Enzymol., 2010, 475, 455-514.

26 R. Roy, S. Hohng and T. Ha, Nat. Methods, 2008, 5, 507-516.

27 A. Katranidis, D. Atta, R. Schlesinger, K. H. Nierhaus, T. CholiPapadopoulou, I. Gregor, M. Gerrits, G. Büldt and J. Fitter, Angew. Chem., Int. Ed., 2009, 48, 1758-1761.

28 K. N. Fish, Curr. Protoc. Cytom., 2009, 12, 12.18.1-12.18.13.

29 D. Magde, W. W. Webb and E. Elson, Phys. Rev. Lett., 1972, 29, $705-$ 708.

30 E. Haustein and P. Schwille, Methods, 2003, 29, 153-166.

31 X. Ren, H. Li, R. W. Clarke, D. A. Alves, L. Ying, D. Klenerman and S. Balasubramanian, J. Am. Chem. Soc., 2006, 128, 4992-5000.

32 S. C. Blanchard, R. L. Gonzalez, H. D. Kim, S. Chu and J. D. Puglisi, Nat. Struct. Mol. Biol., 2004, 11, 1008-1014. 
33 S. Uemura, M. Dorywalska, T. H. Lee, H. D. Kim, J. D. Puglisi and S. Chu, Nature, 2007, 446, 454-457.

34 S. Uemura, R. Iizuka, T. Ueno, Y. Shimizu, H. Taguchi, T. Ueda, J. D. Puglisi and T. Funatsu, Nucleic Acids Res., 2008, 36, e70.

35 D. Kajihara, R. Abe, I. Iijima, C. Komiyama, M. Sisido and T. Hohsaka, Nat. Methods, 2006, 3, 923-929.

36 I. Iijima and T. Hohsaka, ChemBioChem, 2009, 10, 999-1006.

37 E. Boukobza, A. Sonnenfeld and G. Haran, J. Phys. Chem. B, 2001, 105, 12165-12170.

38 E. Rhoades, E. Gussakovsky and G. Haran, Proc. Natl. Acad. Sci. U. S. A., 2003, 100, 3197-3202.

39 B. Okumus, T. J. Wilson, D. M. Lilley and T. Ha, Biophys. J., 2004, 87, 2798-2806.

40 E. Rhoades, M. Cohen, B. Schuler and G. Haran, J. Am. Chem. Soc., 2004, 126, 14686-14687.

41 T. Rosenkranz, A. Katranidis, D. Atta, I. Gregor, J. Enderlein, M. Grzelakowski, P. Rigler, W. Meier and J. Fitter, ChemBioChem, 2009, 10, 702-709.

42 N. Marme, J. P. Knemeyer, M. Sauer and J. Wolfrum, Bioconjugate Chem., 2003, 14, 1133-1139.

43 S. Doose, H. Neuweiler and M. Sauer, ChemPhysChem, 2005, 6, $2277-2285$

44 P. Broz, S. Driamov, J. Ziegler, N. Ben Haim, S. Marsch, W. Meier and P. Hunziker, Nano Lett., 2006, 6, 2349-2353.
45 M. Kumar, M. Grzelakowski, J. Zilles, M. Clark and W. Meier, Proc. Natl. Acad. Sci. U. S. A., 2007, 104, 20719-20724.

46 I. Cisse, B. Okumus, C. Joo and T. Ha, Proc. Natl. Acad. Sci. U. S. A., 2007, 104, 12646-12650.

47 D. Merkle, N. Kahya and P. Schwille, ChemBioChem, 2008, 9, $2673-$ 2681.

48 N. Kahya, Biochim. Biophys. Acta, 2010, 1798, 1392-1398.

49 E. I. Pecheur, D. Hoekstra, J. Sainte-Marie, L. Maurin, A. Bienvenue and J. R. Philippot, Biochemistry, 1997, 36, 3773-3781.

50 N. Kahya, E. I. Pecheur, W. P. de Boeij, D. A. Wiersma and D. Hoekstra, Biophys. J., 2001, 81, 1464-1474.

51 J. Otomo, W. Marwan, D. Oesterhelt, H. Desel and R. Uhl, J. Bacteriol., 1989, 171, 2155-2159.

52 J. Kriegsmann, I. Gregor, d. H. I. von, J. Klare, M. Engelhard, J. Enderlein and J. Fitter, ChemBioChem, 2009, 10, $1823-1829$.

53 J. Kriegsmann, M. Brehs, J. P. Klare, M. Engelhard and J. Fitter, Biochim. Biophys. Acta, Biomembr., 2009, 1788, 522-531.

54 M. J. Levene, J. Korlach, S. W. Turner, M. Foquet, H. G. Craighead and W. W. Webb, Science, 2003, 299, 682-686.

55 J. M. Moran-Mirabal and H. G. Craighead, Methods, 2008, 46, 1117.

56 S. Uemura, C. E. Aitken, J. Korlach, B. A. Flusberg, S. W. Turner and J. D. Puglisi, Nature, 2010, 464, 1012-1017. 